BULL. AUSTRAL. MATH. SOC.

MOS $55 A 30$

VOL. $4(1971), 283-284$.

\title{
Wild knots and arcs in 3-space
}

James M. MePherson

The first four chapters of the thesis are concerned with the development of invariants of local embedding type for certain restricted classes of wild arcs. More particularly, let $M$ be a 3-manifold and $k$ an $\operatorname{arc}$ in $M$ with an isolated wild point $w \in \operatorname{Int} M$ (that is, $w$ has a neighbourhood in $M$ which contains no other wild points of $k$ ). Let $P_{r}$ denote the penetration index of $k$ at $w$, relative to neighbourhoods of $w$ which are "balls with $r$ solid handles". Then if $P_{1} \leq 2$ and $P_{0}>3 P_{1}$, we show in Chapter I that the cofinality class of the knot types of the solid tori occurring in a " $k$-sequence" is an invariant of the non-oriented local arc type of $k$ at $w$.

If $M$ is orientable and $k$ is oriented, $P_{1} \leq 2$ and $P_{0}>3 P_{1}$ as before, we can associate another infinite sequence with $k$ if $k$ satisfies certain rather mild geometric conditions, and we show that the cofinality class of this sequence is an invariant of the oriented local arc type of $k$ at $w$.

Examples are given in Chapter IV to show how these invariants may be used to distinguish arcs in $R^{3}$ or $S^{3}$, whose only wild point is an endpoint (such arcs have been called "nearly polyhedral"). In particular, we exhibit for each odd integer $n \geq 5$ an uncountable family $A_{n}$ of non-invertible nearly polyhedral ares with penetration indices $P_{0}=n$ and $P_{1}=1$ at the point $w$.

Chapter $\mathrm{V}$ relates the geometric invariant of enclosure genus of a wild knot with the algebraic invariant of its nullity. If $M$ is the 3 -sphere

Received 10 November 1970. Thesis submitted to the University of New South Wales, March 1970. Degree approved, July 1970. Supervisor:

Professor N.F. Smythe. 
and $k$ is a knot with one wild point, then the mullity of the Alexander module of $\pi_{1}\left(S^{3}-k\right)$ is bounded above by the enclosure genus of $k$. This result is the best possible, in that for each integer $n$ knots $k_{n}$ and $k_{n}^{\prime}$ are exhibited, both with $P_{0}=2 n$ at their respective wild points, such that $k_{n}$ has enclosure genus and nullity both equal to $n$, while $k_{n}^{\prime}$ has enclosure genus $n$ and nullity 1 .

The technique of "cutting and pasting", used to obtain the results of the first four chapters, is a modification of the technique used by N.F. Smythe ("Isotopy invariants of links", Ph.D. thesis, Princeton University, 1965). The results of Chapter $V$ rest on the fact that the Jacobian module functor preserves colimits. Thus, if $\left\{G_{i}\right\}$ is a family of groups with colimit $G$, and such that for each $i$ there exists an epimorphism $\alpha_{i}$ from $G_{i}$ to a fixed group $H$, then there exists an epimorphism $\alpha: G \rightarrow H$ such that the Jacobian $J H$-module $M[G, \alpha]$ is the colimit of the $J H$-modules $M\left[G_{i}, \alpha_{i}\right]$. 